\title{
The new age of spotted star research using Kepler and CHARA
}

\section{Rachael M. Roettenbacher ${ }^{1}$, John D. Monnier ${ }^{2}$, Robert O. Harmon ${ }^{3}$ and Heidi H. Korhonen ${ }^{4}$}

${ }^{1}$ Department of Astronomy, University of Michigan, 500 Church Street, Ann Arbor, MI, United States email: rmroett@umich.edu

${ }^{2}$ Department of Astronomy, University of Michigan, 500 Church Street, Ann Arbor, MI, United States email: monnier@umich.edu

${ }^{3}$ Department of Physics and Astronomy, Ohio Wesleyan University, 61 S. Sandusky Street, Delaware, OH, United States email: roharmon@owu.edu

${ }^{4}$ Finnish Center for Astronomy with ESO, University of Turku, Väisälänti 20, FI-21500 Piikkiö, Finland email: heidi.h.korhonen@utu.fi

\begin{abstract}
With the precise, nearly-continuous photometry from the Kepler satellite and the sub-milliarcsecond resolving capabilities of the CHARA Array, astronomy is entering a new age for the imaging and understanding of stellar magnetic activity. We present first results from our Guest Observer Program, where 180 single-epoch surface image reconstructions of KIC 5110407 have revealed differential rotation and hints of magnetic activity cycles based on both spot and flare variations. Analysis of our larger, full dataset will establish in unprecedented detail how surface magnetic activity correlates with stellar age and spectral type. In addition to Kepler work, we have harnessed the power of the world's largest infrared interferometer to "directly" image the spotted surfaces of a few of the closest RS CVn systems, allowing a comparison of contemporaneous Doppler and light-curve inversion imaging techniques.
\end{abstract}

Keywords. stars: spots, stars: activity, stars: imaging, stars: low-mass

\section{Introduction}

Starspots, analogous to sunspots, are areas of the stellar photosphere that are significantly cooler than their surroundings, appearing darker. In hot stars, these spots are regions of metals accumulating near the magnetic poles (e.g. Krtička et al. 2007). In cool stars, these spots are due to strong magnetic fields quenching convection in the outer layers of the atmosphere. Because these spots are the clearest manifestation of magnetic fields their structure and evolution is important to understanding stellar magnetic dynamos. Tracing spot motions over time allows for observations of spot evolution and measurements of differential rotation, important concepts in the understanding stellar activity and magnetic behavior (Strassmeier 2009).

To better understand starspots, we aim push the boundaries of current imaging techniques in order to study surface spot structures and evolution. We compare several stateof-the-art imaging techniques to validate methods and compare results. Additionally, we use one of these imaging techniques for analyzing stars with rapidly-evolving spot structures using the nearly-continuous precision photometry of the Kepler satellite. 


\section{Starspot Imaging}

\subsection{Long-baseline Interferometry}

Starspots have been imaged with a variety of techniques involving observations obtained via interferometry, spectroscopy, and photometry. With interferometry, the starspots of bright, nearby stars have begun to be observed (e.g. Parks et al. 2011). With the recently upgraded Michigan Infrared Combiner (MIRC) at Georgia State University's Center for High Angular Resolution Astronomy (CHARA) Array, it is now possible to use all six CHARA telescopes to directly image stars with spotted surfaces via aperture synthesis modeling. Stars to be imaged interferometrically are required to be bright and have large angular diameters. Spectroscopically, Doppler imaging allows for reconstructions of surface features based upon variations of absorption line profiles as the star rotates (e.g. Vogt \& Penrod 1983). A method that can only be applied to rapidly-rotating stars, Doppler imaging requires high-resolution spectroscopy to trace the motions of spots across a stellar surface through changing spectral features. Photometric light-curve inversion techniques allow for reconstructions based upon flux variations (e.g. Roettenbacher et al. 2011). We aim to image stars simultaneously with all three of these techniques to compare the results and better understand the surface features of spotted stars.

Because each technique has strict requirements for targets our sample is limited. Four observable spotted star candidates have been identified: $\zeta$ And, $\sigma$ Gem, $\epsilon$ UMa, and $o$ Dra. $o$ Dra, $\zeta$ And, and $\sigma$ Gem are all the primary stars of RS CVn systems and known to be active and periodically variable, suggesting spot activity (e.g. Wilson 1976, Gratton 1950, and Eberhard \& Schwarzschild 1913, respectively). $\epsilon$ UMa is a chemically peculiar Ap star with periodic light curve variations (e.g. Struve \& Hiltner 1943). In the first study of its type, we have successfully coordinated interferometric observations with high-resolution spectroscopy and photometry targeting these stars. We aim to compare the aperture synthesis imaging using interferometric data with the results of spectroscopic Doppler imaging and photometric light curve inversion. To date, spotted stellar surfaces have been mapped with each technique, but the three techniques have not been verified against one another with simultaneous data.

For our observations, we obtain data from a variety of sources. The interferometric data are all collected at the CHARA Array with MIRC. Doppler imaging reconstructions for this work are obtained at the European Southern Observatory's Very Large Telescope (VLT), the Nordic Optical Telescope (NOT), the STELLA robotic telescope, and the Belgian Mercator telescope. The photometric data are obtained by the Tennessee State University 0.4-m Automated Photometric Telescope (APT) at Fairborn Observatory, AZ and the SMARTS 1.3-m telescope at Cerro Tololo, Chile.

Preliminary analyses of our data sets show no evidence of spots in the interferometric data for $\epsilon$ UMa and $o$ Dra, and our $\sigma$ Gem data were plagued by bad weather at CHARA. A very preliminary study of $\zeta$ And from 2011 July indicates the consistency between the Doppler imaging and the interferometric aperture synthesis mapping. We hope to compare the 2011 data to the our observing campaign of $\zeta$ And occurring during 2013 September and October. While directly comparing the results of the three image reconstructions for the first time, we will also be able to identify significant changes in the stellar surface over the course of two years.

\subsection{High-cadence Photometry}

In addition to the simultaneous imaging techniques of select spotted stars, we analyze the light curve of a population of stars with rapidly-changing spot features to characterize spot evolution and differential rotation observed by the Kepler satellite. The advantage 


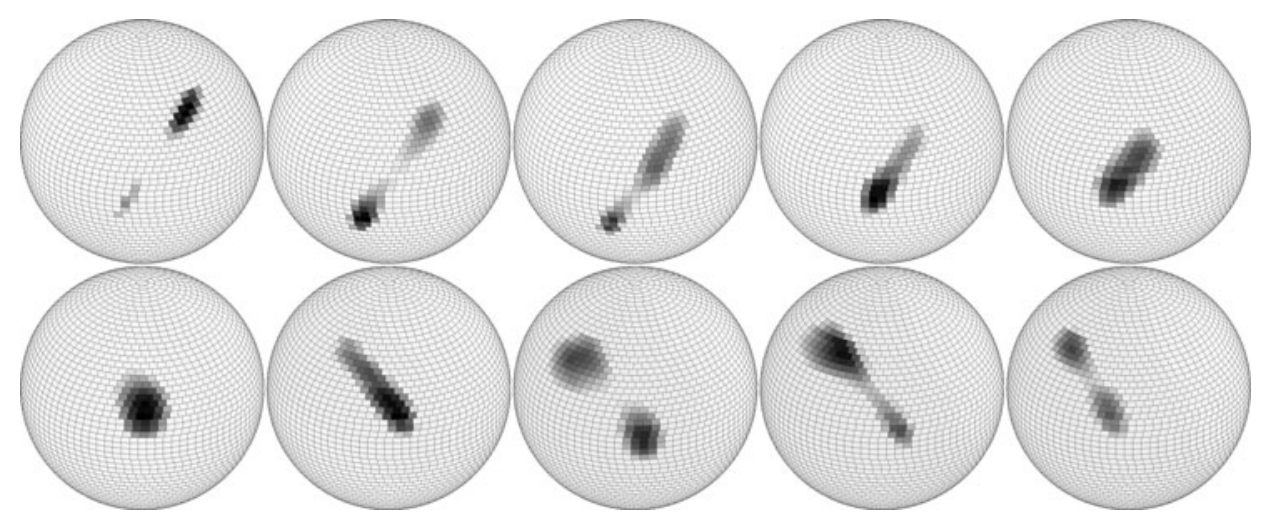

Figure 1. Panel of ten reconstructed surfaces of KIC 5110407 with an inclination of $i=60^{\circ}$. Each sequential surface (starting in the upper left with the first data point at BJD 2455124.43) is centered on the same latitude and longitude to illustrate spot evolution from one rotation period to the next (plot reprinted from Roettenbacher et al. 2013).

of photometry from the Kepler satellite is the the high-precision, nearly-continuous data of 30-minute cadence in comparison to ground-based observations.

We utilize a constrained, non-linear inversion algorithm, Light-curve Inversion (LI; see Harmon \& Crews 2000), for mapping starspots from the Kepler photometric light curves. LI makes few a priori assumptions about the spotted stellar surface. No assumptions are made on spot shape, number, or size. Estimates for spot and photospheric temperatures, limb darkening coefficients, and rotation rate are made based on published values and the results of our analyses. LI has been tested extensively with simulations (Harmon \& Crews 2000) and used for ground-based photometry (including comparison to Doppler images at some epochs; Roettenbacher et al. 2011) and for space-based photometry (Roettenbacher et al. 2013).

As a preliminary study and test of the method, we have applied LI to the light curve of a star in the Kepler field, KIC 5110407, a K-type pre-main sequence candidate that exhibits rapidly-changing spot structures. Our inverted surfaces show spot evolution and differential rotation (see Figure 1). We quantified the stellar differential rotation using the differential rotation law

$$
\Omega(\theta)=\Omega_{\mathrm{eq}}\left(1+k \sin ^{2} \theta\right)
$$

where $\Omega(\theta)$ is the angular velocity as a function of latitude, $\Omega_{\mathrm{eq}}$ is the angular velocity at the equator, $\theta$ is spot latitude, and $k$ is the differential rotation parameter (as in Henry et al. 1995). Regardless of inclination, we found a value of $k$ to be less than a scaled solar model (see Figure 2), consistent with stellar dynamo theories (e.g. Hall 1991).

With KIC 5110407 and other stars in our Kepler program, we aim to quantify differential rotation as well as analyze the presence of short-period activity cycles. For example, we saw evidence in the flares of KIC 5110407 to indicate the possible presence of an activity cycle. In the data (Quarters 2-5 and 7-9), we identified seventeen flares. We found an unusual concentration of flares between Q4-5, and a period without any flares in Q7-9 lasting over 200 days. By repeating our analysis of KIC 5110407 on other spotted stars in the Kepler field, we will be able to identify activity cycles, furthering our knowledge of the mechanisms of spot activity. With an understanding of activity cycles on a variety of stars, we hope to establish a relationship between stellar age and spectral type. 


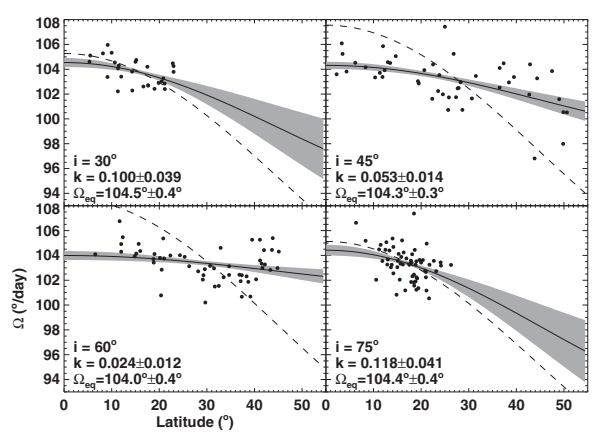

Figure 2. Derived differential rotation parameter, $k$, varies with the assumed angle of inclination, highlighting the degeneracy of the solutions of Light-curve Inversion (LI). KIC 5110407 has sub-solar differential rotation (solid line with $1-\sigma$ errors in gray; fit with the solar $k$ value, dashed line; plot reprinted from Roettenbacher et al. 2013).

\section{Summary and Future Work}

We are working on a campaign to cross-compare and validate several state-of-the-art imaging methods. By obtaining simultaneous interferometric, spectroscopic, and photometric data we will be able to directly compare reconstructed images of spotted stellar surfaces. In the first study of this type, we aim to obtain data on four spotted stars. The most promising data set will be obtained in 2013 September and October on the known spotted RS CVn $\zeta$ And, combining many resources to ensure the best temporal coverage for directly comparing images from different techniques and data sets.

While comparing three imaging methods, we are also focusing efforts on reconstructing spotted surfaces from the precision photometry of the Kepler satellite. Our first results on Kepler star KIC 5110407 show evidence of spot evolution, differential rotation, and potential evidence of an activity cycle (Roettenbacher et al. 2013). We will apply the techniques used in our test case to many other stars with rapidly-evolving spots with the ultimate goal of establishing a relationship between stellar age and spectral type based upon activity.

\section{References}

Eberhard, G. \& Schwarzschild, K. 1913, ApJ, 38, 292

Gratton, L. 1950, ApJ, 111, 31

Hall, D. S.1991, in The Sun and Cool Stars: Activity, Magnetism, Dynamos, ed. I. Tuominen,

D. Moss, \& G Rüdiger (Lecture Notes in Physics, Vol. 380; Berlin: Springer), 353

Harmon, R. O. \& Crews, L. J. 2000, AJ, 120, 3274

Henry, G. W., Eaton, J. A., Hamer, J., \& Hall, D. S. 1995, ApJS, 97, 513

Krtička, J., Mikulášek, Z., Zverko, J., \& Žižňovský, J. 2007, A $\& A, 470,1089$

Parks, J. R., White, R. J., Schaefer, G. H., Monnier, J. D., \& Henry, G. W. 2011, in ASP Conf. Ser. 448, 16th Cambridge Workshop on Cool Stars, Stellar Systems, and the Sun, ed. C. M. Johns-Krull, M. K. Browning, \& A. A. West (San Francisco, CA: ASP), 1217

Roettenbacher, R. M., Harmon, R. O., Vutisalchavakul, N., \& Henry, G. W. 2011, AJ, 141, 138

Roettenbacher, R. M., Monnier, J. D., Harmon, R. O., Barclay, T., \& Still, M. 2013, ApJ, 767,60

Strassmeier, K. G. 2009, A\& ARv, 17, 251

Struve, O. \& Hiltner, W. A. 1943, ApJ, 98, 225

Vogt, S. S. \& Penrod, G. D. 1983, PASP, 95, 565

Wilson, O. C. 1976, ApJ, 205, 823 